On the "massless gap" adjustment of detected energy for passive material in front of a calorimeter ${ }^{\star}$

\title{
HANS-Jochen Trost
}

High Energy Physics Division - 362 -

Argonne National Laboratory

9700 S. Cass Ave., Argonne, IL 60499-4815, USA

31 January 1992

\section{ABSTRACT}

I have designed a correction scheme for energy losses in passive material in front of a calorimeter based on the "massless gap" idea. I use a flexible geometry model of a calorimeter design for SDC outside of a solenoidal coil made of aluminium cylinders of adjustable thickness. The signal from the first radiation length of active calorimetry is scaled dependent on the incoming and observed energies of the shower. A reasonable recovery of the resolution of an unobstructed calorimeter is achieved using correction factors that depend only upon the total thickness of passive material. Thus a useful correction may be built into the hardware by increasing the amount of scintillator in the first radiation length of the active calorimeter. The distribution of correction factors determined eventby-event indicate that an additional dependence on the observed signal in the massless gap and total incident energy is clearly present.

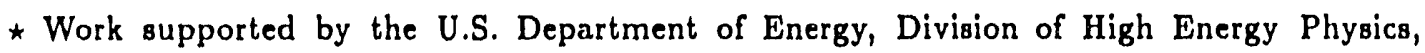
Contract W-31-109-ENG-38 


\section{Introduction}

The accurate measurement of particle and jet energies is one of the central performance goals for designing and constructing the SDC detector. ${ }^{[1]}$ A recurring issue in this context is the amount of material in front of the active calorimetry. ${ }^{[2-6]}$ One method to accomodate passive material is to install "massless gaps", some active element, into or just behind the passive material to turn it into a more or less small sampling calorimeter on its own. With the solenoidal coil and tracking systems in front, the SDC calorimeter ${ }^{[1]}$ has to cope with this kind of a problem to some degree. I have chosen to use the ANLSIM simulation program ${ }^{[10]}$ with the a flexible geometry model ${ }^{[1]}$ to design and test a correction scheme of the massless gap variety for relatively localized passive material, like the solenoidal coil and calorimeter shell materials, in front of the calorimeter for showers from single electrons at energies of 10,20 , and $50 \mathrm{GeV}$.

\section{Detector setup and analysis method}

The detector model used in the present study has been described in detail before. ${ }^{[5]}$ For my present purpose, I point single particle beams at $90^{\circ}$ into the barrel calorimeter (Fig.1) and vary the thickness of the central cylinder in the coil model to obtain the desired round amounts of passive material $(1,2,3$ and 5 radiation lengths). In the program, the calorimeter is represented by a material mixture appropriate for a sandwiching of $4 \mathrm{~mm}$ thick lead absorbers and $4 \mathrm{~mm}$ thick scintillator tiles through the full depth of the calorimeter, with no additions for air gaps, wrappings, readout cracks or any mechanical support members. To calibrate the calorimeter response, I also made runs without any coil, i.e. the beam traverses $2.25 \mathrm{~m}$ of air, filled with an axial homogeneous magnetic field of 2 Tesla strength out to a radius of $1.7 \mathrm{~m}$, before reaching the calorimeter. Thus, this study covers the relevant range for the scintillator-tile calorimeter design which sees at $90^{\circ}$ a coil equivalent to $9.54 \mathrm{~cm}$ of aluminium ${ }^{(12)}$ and a shell of $1 / 8$ inch steel, corresponding to a total of 1.25 radiation lengths, and 
possibly around half a radiation length from the tracking system in front of its sensitive region; at about $26^{\circ}$, near the barrel-endcap transition, these numbers increase to 2.86 plus about 1 radiation lengths. There is, in some designs for the electromagnetic section, a gap of about $8 \mathrm{~cm}$ between the barrel and the endcap, corresponding to $\Delta \eta \approx 0.04$. Requiring full shower containment, the gap effectively doubles leaving me with full shower acceptance over $96 \%$ of the rapidity range $|\eta| \leq 2.5$. To study the impact of the nonvertical angle of incidence on the resolution, I perform calculations with the 1.25 radiation lengths thick coil I have used before ${ }^{[b]}$ at a few polar angles from $90^{\circ}$ down to $30^{\circ}$. (This means stopping just short of the gap between the barrel and endcap because the gap is different in my model from the one in the current baseline design.) I do not include a tracking system model because that system will supposedly deliver the reference energies for measuring the correction in situ.

In the present study, I let ANLSIM and GEANT follow all particles in analog mode down to kinetic energies of $100 \mathrm{keV}$ except photons which are taken down to $10 \mathrm{keV}$. Point deposits of energy created during the analog shower propagation are smeared with gamma distributions $\gamma(x ; k, 1.0)$, with a variance of $k=0.15^{2} \mathrm{GeV} / E_{\text {deposit }}$, to give the overall simulation a consistent resolution behavior. The choice of $\gamma$ distributions avoids the large negative tail of the smeared distribution of deposited energy which appear for a Gaussian of the same variance at the small energies of the deposits I have to deal with; the accumulated distributions for showers in the $\mathrm{GeV}$ energy range receive proper Gaussian-like shapes in this approach. For $\gamma(x ; k, 1.0)$ distributions, the variance is equal to the mean, $k$. The algorithms for random number generation are taken from the Review of Particle Properties book ${ }^{[13)}$ and a paper by Ahrens and Dieter. ${ }^{[1, !)}$ The values of $k$ requested by the simulation fall mostly into the interval $k=10^{-3}-10^{-1}$, distributed nearly flat over $\log _{10} k$ in the specified range. At $k=10^{-3}$, about $95.5 \%$ of the integral of the $\gamma(x ; k, 1.0)$ distribution is located at $x<10^{-20}$. This property delivers effectively a simulation of the sampling fraction of the calorimeter, rendering this technique appropriate for the present purpose. By comparing 
program runs done at different times during the evolution of the present study, I find an uncertainty arising from the particular implementation of the gamma distributions in the measurement of the mean energy over the full event samples of about $1 \%$ which is much more than the statistical error of that mean. The resolutions receive from the same source an uncertainty of about $8 \%$ (relative).

The massless gap is chosen to be approximately the first radiation length of the active calorimeter, i.e. $8 \mathrm{~mm}$ in the coded detector out of a radial thickness of active calorimetry at $90^{\circ}$ of $200.0 \mathrm{~cm}$. This is the only type of longitudinal segmentation I use. Due to the approximation of having a continuous, totally active calorimeter, this corresponds most closely to a design in which a scintillator layer in front of the first absorber layer and a scintillator right behind the first absorber layer are both read out together. The transverse segmentation is a standard one, about $0.05 \cdot 0.05$ in the $(\eta, \phi)$ plane. I form clusters using seed cells containing at least $1 \mathrm{GeV}$ of energy and collect all cells with more than $3 \mathrm{MeV}(!)$ within a radius of $R=0.5$ in the $(\eta, \phi)$ plane. If more than one cluster is found, I look only at the most energetic one. This choice eliminates all backscattered secondary particles that hit the calorimeter far away from the primary electron; obvious examples are contained in Fig.1.

Calling the signal from the first radiation length $E_{\text {front }}$, and the signal from the remainder of the calorimeter $E_{b a c k}$, I write the measured energy

$$
E_{\text {corr }}=\alpha_{m g} \cdot E_{\text {front }}+E_{\text {back }}
$$

as has been used in refs.6-8. This equation can be inverted as

$$
\alpha_{m g}=\left(E_{\text {corr }}-E_{\text {back }}\right) / E_{\text {front }}
$$

if the correct energy $E_{\text {corr }}$ is known. I use the measured mean energy from the program runs without any coil for this purpose, and calculate the correction factor on an event-by-event basis instead of from the mean values over all simulated 
events. This allows me to measure the effect of fluctuations on the correction factor and the dependences both on the signal of the massless gap and the beam energy.

By design, the simulation represents a realistic data taking procedure, and the subsequent analysis is without modification suitable for application to an experimental approach of measuring the massless-gap correction; the desired nominal energy would there be supplied to sufficient accuracy by a magnetic measurement of the particle momentum. For electrons, even a measurement in the SSC interaction region is conceivable by selecting a sample of events containing clean decays $Z^{0} \rightarrow e^{+} e^{-}$as long as the resolution of the tracking system is noticeably better than the calorimeter resolution: The electrons can be defined by matching charged tracks with electromagnetic clusters without being limited severely by their relative and absolute inaccuracy, with the additional condition of having an $e^{+} e^{-}$pair near the $Z^{0}$ mass. Then the central detector will deliver a measurement of the desired energy $E_{\text {corr }}$, and a suitably designed calorimeter with a massless gap will provide the other two data elements, $E_{\text {front }}$ and $E_{\text {back }}$. In the case of the SDC detector, the expected resolution of the tracking system ${ }^{[1]}$ of $\sigma_{p_{t}} / p_{t}=0.25(\mathrm{TeV} / \mathrm{c})^{-1} \cdot p_{t}$ is fairly limited as at a momentum of $75 \mathrm{GeV} / \mathrm{c}$ at $90^{\circ}$ one has only $\sigma_{p} / p \approx \sigma_{E} / E \approx 1.8 \%$. This in situ determination will therefore work in the SDC detector only for energies below about $50 \mathrm{GeV}$ (or less), i.e. a quite limited range in pseudorapidity and transverse momentum.

\section{Analysis and results}

I generate 500 events for every beam energy and coil thickness, except at $50 \mathrm{GeV}$ where I have about 450 to 460 events each. For my reference case, the calorimeter model without any material in front of it (except for the $2.25 \mathrm{~m}$ of air), the mean energy response turns out to be very well linear. The resolution of $\sigma_{E} / E=0.15 / \sqrt{E} \mathrm{I}$ have used to fluctuate the individual energy deposits is maintained in the total signal; this choice matches the expectation from experi- 
mental data (Fig.2). The case without passive material provides for a good cross check for the determination of the correction factor $\alpha_{m g}$ : As there is nothing to correct for, it has to come out as $\alpha_{m g}=1.0$ on average, and the fluctuations in its determination event-by-event reflect the fluctuations in the energy measurements in the front and back sections with a variance of

$$
\begin{aligned}
\left(\sigma_{\alpha}\right)^{2} & =\frac{\left(\alpha_{m g} \sigma_{\text {front }}\right)^{2}+\left(\sigma_{\text {back }}\right)^{2}}{E_{\text {front }}^{2}} \\
& \approx\left(\frac{\sigma_{\text {back }}}{E_{\text {front }}}\right)^{2} \quad \text { for } \sigma_{\text {back }} \gg \sigma_{\text {front }}
\end{aligned}
$$

(derived from eq.(2.2)). Taking as an example the mean and r.m.s. values for electrons at $20 \mathrm{GeV} / \mathrm{c}$,

$$
\begin{aligned}
E_{\text {corr }} & =19.95 \mathrm{GeV} \\
E_{\text {back }} & =19.91 \mathrm{GeV} \\
E_{\text {front }} & =0.0264 \mathrm{GeV} \\
\sigma_{\text {back }} & =0.715 \mathrm{GeV} \\
\sigma_{\text {front }} & =0.0275 \mathrm{GeV} \\
<\alpha_{m g}> & =1.50,
\end{aligned}
$$

I obtain

$$
\sigma_{\alpha} \approx 27.1
$$

almost independent of $\alpha_{m g}$, from eq.(3.1) which compares very well to the actual distribution of the correction factors measured in the simulation run (Fig. 3 ; this is true also for the other energies investigated). With the statistics available, the expected "measurement" error for the mean $\alpha_{m g}=1.0$ is $\Delta \alpha_{m g} \approx 1.2$. This sets the scale at which I can claim significance for the need of a correction.

Considering now material in front of the calorimeter, in totals of one, two, three and five radiation lengths of aluminimum in a coil arrangement (Fig.1), I find a clear peak developing in the distribution of the correction factor $\alpha_{m g}$, with 
a part of the long tails from the shower fluctuations still present in the case of only one radiation length of material (Fig.3). The mean value of the peak is a few for the larger amounts of material, in agreement with the results previously obtained $^{(7)}$ for the SDC liquid argon calorimeter design. These "well-defined" correction factors appear actually as soon as the detected energy in the massless gap exceeds the average size it has with no material in front. This becomes obvious in the scatterplots of $\alpha_{m g}$ versus the energy $E_{\text {front }}$ as well as versus the ratio $E_{\text {front }} / E_{b a c k}$ (Figs.4,5). At this point, a possible choice for performing a correction is to use the mean value of the event-by-event determined factors $(\text { Tab. } 1)^{\star}$. This improves the raw resolutions (for more than one radiation length of passive material) already quite significantly (Tab.2, second column). Picking by hand some near-average values independent of the beam energy I can retain most of the improvement. This means that increasing the signal output from the massless gap by those energy-independent factors for the appropriate amount of passive material in front, this part of the best possible correction can be built into the detector, leaving a smaller correction to be done by software afterwards. For only one radiation length of passive material in front of the calorimeter, the deterioration effects are small; correspondingly the corrections are still quite badly defined and do not deliver any noticeable improvement. All of this agrees well with the recent study done by Barry Wicklund. ${ }^{[0]}$

With the good statistics I have available, I go one step further and look at a correlation between $\alpha_{m g}$ and the gap energy or energy ratio. By averaging the $\alpha_{m g}$ values for small bins of the gap energy or energy ratio, I find a clear dependence of the best correction factor on the energy observed in the massless gap (Figs.6,7) for the larger amounts of passive material. I fit these distributions

\footnotetext{
$\star$ Actually, the means are determined by fitting Gaussian curves to the central parts of the distributions. The resulting factors are mostly only a little smaller than the directly calculable unrestricted means of the distributions. They are better determined in all cases and exhibit a more reasonable variation with energy and the thickness of passive material. The resulting resolutions do not deviate much from those obtained with the unrestricted means. In particular, the unrestricted means do not fully restore the linearity of the calorimeter response, a topic discussed further below.
} 
with an exponential function plus a constant. Cornparing the use of the front energy or the front-to-back ratio as a parameter, I observe here that the front energy seems to give a little better defined functions than the ratio and choose therefore to use the front energy as a parameter for the determination of all my corrections. Applying these functions does not improve the resolutions noticeably over the constant factor corrected results. This is likely due to the still limited statistics in particular for the smallest signals from the massless gap. In some cases, foremost the angular dependence at $50 \mathrm{GeV}$, a sensible determination of such functions is even impossible.

To have a useful correction function for the final experiment, I need to interpolate between the chosen energies where I have determined the correction. For this purpose, I look at the dependence of the coefficients both on the energy and the thickness of the passive material with the added boundary condition that $\alpha_{m g} \rightarrow 1.0$ in either limit of high particle energy and vanishing material thickness. The following parametrization

$$
\begin{aligned}
\alpha_{m g}(d, E) & =1.0+a_{1} \cdot d+a_{2} \cdot e^{-a_{3} \cdot E} \\
a_{1} & =0.7729 \\
a_{2} & =3.888 \\
a_{3} & =0.14435
\end{aligned}
$$

with $d$ being the thickness of the passive material in units of radiation length, $X_{0}$, and $E$ the incident energy in $G e V$, turns out to give a good final result, not optimized but as good as, and in the angular dependence for the SDC coil even better than, the correction with the constant factors (Tab.2). The coefficients $a_{i}$ are determined from crudely fitting the individual factors in the table with the function of eq.(3.3). For nonvertical angles of incidence, I take out the $1 / \sin \theta$ facotr of increase in the thickness of the massless gap effect by multiplication,

$$
\alpha_{m g}(\theta)=\alpha_{m g}\left(90^{\circ}\right) \cdot \sin \theta
$$

(Note that the geometry as chosen here gives a signal enhancement just due to 
this factor as a constant thickness of the massless gap transversely to the beam axis is assumed.) The passive material thickness still has to be taken along the direction of the incident electron, not transversely to the beam. 'The resulting resolutions are shown in Fig.8. (The previously roted uncertainties inherent in the simulation are responsible for the scattering of the points.)

Beyond the deterioration of the resolution, I also have to look at the behavior of the mean response (Tab.3). Although in first approximation the reduced response is "calibrated away", such a calibration is an expression of the mere fact that the passive material in front of the calorimeter gives rise to a nonlinearity in the response in an energy region of high importance for achieving the physics goals of the SDC experiment, e.g. the measurements of the masses of the $Z^{0}$, or through it of the neutral Higgs boson, or of the top quark using pairs of an isolated electron with a nonisolated muon.! (Remember that, as mentioned before, at energies beyond about $50 \mathrm{GeV}$ the tracking system will not any more outperform the calorimeter on resolution.) On this front I find that the corrections do in fact reduce also the nonlinearity significantly, with the overall funtional correction again giving the best results. As a bottom line both on the resolution and the linearity issues, for the amounts of passive material to be dealt with in the scintillator-tile calorimeter of SDC, the corrections from a massless gap are fairly small at the center of the barrel calorimeter (compared to e.g. a liquid argon calorimeter ${ }^{[0-8]}$ ), increasing somewhat towards the endcap regions due to the $1 / \sin \theta$ effect. A constant enhancement factor along the barrel, independent of energy, does already improve both the resolution and linearity although without removing the need for further corrections to be done after data taking. On this basis, an extensive study of the final calorimeter design will be needed to establish the particular technique of setting up a massless gap. This is beyond the scope of the present work. 


\section{Summary}

I have studied a massless gap approach to correcting the calorimeter response to electromagnetic showers for passive material in front of the calorimeter. I find that at two radiation lengths of passive material, the correction using this approach becomes worthwhile, delivering a noticeable improvement. A part of the correction can be built into the detector by designing for an appropriately enhanced signal from the massless gap. Beyond the recovery of the resolution, the nonlinearity introduced by the passive material is also noticeably eased.

\section{ACKNOWLEDGEMENTS}

I gratefully acknowledge the interest and contributions of Dr. Tom Kirk who not only brought the whole issue to my attention, thereby initiating this study, but also supported its development throughout with his comments, suggestions and criticism. I also thank Dr. Chris Hearty for his opinions and ideas, and Dr. David Malon for his support and consulting in getting a handle on the gamma distributions for my programs.

\section{DISCLAIMER}

This report was prepared as an account of work sponsored by an agency of the United States Government. Neither the United States Government nor any agency thereof, nor any of their employees, makes any warranty, express or implied, or assumes any legal liability or responsibility for the accuracy, completeness, or usefulness of any information, apparatus, product, or process disclosed, or represents that its use would not infringe privately owned rights. Reference herein to any specific commercial product, process, or service by trade name, trademark, manufacturer, or otherwise does not necessarily constitute or imply its endorsement, recommendation, or favoring by the United States Government or any agency thereof. The views and opinions of authors expressed herein do not necessarily state or reflect those of the United States Government or any agency thereof. 


\section{REFERENCES}

1) Solenoid Detector Collaboration, G.H.Trilling et al., SDC-90-00085 and SSC-EOI-3, SSC Laboratory, Dallas, Texas, May 24, 1990, and SDC-90-00151 and SSC-LOI-1, SSC Laboratory, Dallas, Texas, November 30,1990

2) A.M.Jonckheere, Simulating DO and Hermeticity Studies or How to Effect a Detector Design, Proc. Workshop on Detector Simulation for the SSC, August 24-28, 1987, ed. L.E.Price, ANL-HEP-CP-88-51, Argonne National Laboratory, Argonne, Illinois, USA 1988, p.270, p.175

3) M.Strovink, W.J.Womersley and G.E.Forden, Hermeticity of three Cryogenic Calorimeter Geometries, Proceedings of the Workshop on Calorimetry for the Supercollider, March 13-17, 1989, Tuscaloosa, Alabama, U.S.A., eds. R.Donaldson and M.D.G.Gilchriese, World Scientific Publishing Co. Pte. Ltd., Singapore, 1990, p.9

4) R.Ruchti, Summary Report for the Scintillating Fiber Working Group, Proceedings of the Workshop on Tracking Systems for the Superconducting Supercollider, July 24-28, 1989, TRIUMF Laboratory, Vancouver, Canada, 1989, E3

5) T.B.W.Kirk and H.-J.Trost, The effect of passive material on the detection of hadrons in calorimeter configurations for the SDC detector, ANL/HEP/TR-91/62 and SDC-91-00050

6) H.Hirayama, Effects of dead material to the electro-magnetic calorimeter and energy/resolution recovery with "massless gap", JSD-NOTE-199030 and SDC-90-00108, Nucl.Instr.Meth. A302 (1991) 427

7) Ch.Hearty, Resolution and hermeticity of the liquid argon electromagnetic calorimeter as a function of pseudorapidity, SDC-90-00145-REV 
8) SDC Liquid Argon Group, Calorimeter conceptual design - liquid argon option, 5 August 1991 (uncatalogued)

9) A.B.Wicklund, EGS4 studies of CDF preshower response: B-field effects and "massless gap" resolution response, CDF/ANAL/ELECTRON /PUB/1639, 2 January 1992

10) R.E.Blair, L.E.Price and H.-J.Trost, program ANLSIM, Argonne National Laboratory, Argonne, Illinois, USA 1989, version 1.05, 1991 (unpublished);

H.-J.Trost, ANLSIM, talk given at: Workshop on the Future Development of GEANT, SSC Laboratory, Dallas, Texas, Jan.10-13, 1990, unpublished;

R.E.Blair, The Argonne National Laboratory Simulation Package, Proceedings of the Workshop of Physics and Detector Simulation for SSC Experiments, SSC Laboratory, Dallas, Texas, January 9-19, 1990, vol.1, p.69;

H.-J.Trost, How to Use ANLSIM, ibid., vol.2, p.265

11) J.Proudfoot and H.-J.Trost, A first simulation study of the barrel-endcap transition region in a calorimeter of the scintillator tile design, ANLHEP-TR-90-77 and SDC-90-00073

12) R.Fast et al., SDC Calorimeter Group Meeting at Fermilab, May 21-22, 1991

13) Particle Data Group, Review of Particle Properties, Phys.Tett. 239B (1990), p.III.38, algorithm for $k>1$; note that for the second parameter not equals to 1.0 as well as for $k<1$, the algorithms don't work as given there.

14) J.H.Ahrens and U.Dieter, Computing 12 (1974) 223, algorithm GS for $k<1$ 


\section{TABLE CAPTIONS}

1. Correction factors: individual mean factors, energy independent factors

2. Resolutions for different massless gap correction schemes: no correction, individual mean, energy independent mean, individual function, overall function

3. Mean responses for different massless gap correction schemes: no correction, individual mean; anergy independent mean, individual function, overall function

\section{FIGURE CAPTIONS}

1. Calorimeter and coil model ( $2 X_{0}$ version) used in ANLSIM for the present study. The shower included is due to an electron of $20 \mathrm{GeV} / \mathrm{c}$ momentum (the same event in the overview and the enlarged view), and particle trajectories are shown for kinetic energies beyond $10 \mathrm{MeV}$.

2. Mean response and resolution over incident energy for electrons in the simulated calorimeter

3. Event-by-event determined correction factor $\alpha_{m g}$ for electrons with a momentum of $20 \mathrm{GeV} / \mathrm{c}, \mathrm{a})$ with no passive material in front of the calorimeter, b) with $1 X_{0}$ of aluminium in front, c) with $2 X_{0}$, d) with $\left.3 X_{0}, \mathrm{e}\right)$ with $5 X_{0}$. The distributions are fit with a single Gaussian in the range $\alpha_{m g}=-25.0 \rightarrow+25.0$.

4. Distribution of $\alpha_{m g}$ versus the detected gap energy $E_{\text {front }}$, for electrons at $20 \mathrm{GeV} / \mathrm{c}$; cases as in Fig.3

5. Distribution of $\alpha_{m g}$ versus the ratio of energies $E_{\text {front }} / E_{b a c k}$, for electrons at $20 \mathrm{GeV} / \mathrm{c}$; cases as in Fig.3 
6. Dependence of $\alpha_{m g}$ on the gap energy $E_{\text {front }}$, for electrons at $20 \mathrm{GeV} / \mathrm{c}$; cases as in Fig.3

7. Dependence of $\alpha_{m g}$ on the energy ratio $E_{\text {front }} / E_{b a c k}$, for electrons at $20 \mathrm{GeV} / \mathrm{c}$; cases as in Fig.3

8. Resolution dependence on a) the thickness of passive material and b) the angle of incidence into the coil and calorimeter 
Table 1a: Mean correction factors for different electron energies and thicknesses of passive material

\begin{tabular}{ccccc} 
& $10 \mathrm{GeV}$ & $20 \mathrm{GeV}$ & $50 \mathrm{GeV}$ & $\begin{array}{c}\text { energy } \\
\text { independent }\end{array}$ \\
\hline $0 X_{0}$ & -0.1755 & 1.502 & -1.096 & 1.0 \\
$1 X_{0}$ & 2.638 & 0.1564 & 0.3843 & 1.1 \\
$2 X_{0}$ & 3.006 & 2.337 & 1.913 & 2.2 \\
$3 X_{0}$ & 4.408 & 3.595 & 3.127 & 3.3 \\
$5 X_{0}$ & 6.381 & 5.510 & 4.491 & 5.5 \\
\hline
\end{tabular}

Table $1 b$ : Mean correction factors for different electron energies and angles of incidence into the SDC coil

$$
10 \mathrm{GeV} \quad 20 \mathrm{GeV} \quad 50 \mathrm{GeV} \begin{gathered}
\text { energy } \\
\text { independent }
\end{gathered}
$$

\begin{tabular}{lllll}
\hline $90^{\circ}$ & 1.018 & 1.101 & 1.089 & 1.4 \\
$45^{\circ}$ & 1.230 & 1.266 & 1.122 & 1.4 \\
$35^{\circ}$ & 1.255 & 1.231 & 1.033 & 1.4 \\
$30^{\circ}$ & 1.292 & 1.239 & 0.9787 & 1.4 \\
\hline
\end{tabular}

The correction factor is changed to 1.0 for $0 X_{0}$ always (nothing to correct) and for $\alpha_{m g}<1.0$ (values less than 1.0 do not make sense, and their errors are still larger than 1.0). 
Table 2a: Resolutions (in $\mathrm{GeV}$ ) for different massless-gap correction schemes:

Dependence on thickness of passive material

$$
\begin{array}{cccc}
\text { no correction } & \text { individual individual } & \text { energy } & \text { overall } \\
\text { mean } & \text { function independent function }
\end{array}
$$

\begin{tabular}{llllll}
\hline $10 \mathrm{GeV}$ & & & & & \\
$0 X_{0}$ & 0.4975 & & & & \\
$1 X_{0}$ & 0.4633 & 0.4882 & 0.4803 & 0.4656 & 0.4867 \\
$2 X_{0}$ & 0.5356 & 0.5296 & 0.5291 & 0.5280 & 0.5618 \\
$3 X_{0}$ & 0.6322 & 0.6366 & 0.6131 & 0.5904 & 0.6313 \\
$5 X_{0}$ & 0.9567 & 0.8978 & 0.9826 & 0.8912 & 0.8876
\end{tabular}

\begin{tabular}{cccccc}
$20 \mathrm{GeV}$ & & & & & \\
$0 X_{0}$ & 0.6790 & & & & \\
$1 X_{0}$ & 0.7001 & $(0.7001)$ & 0.6834 & 0.7008 & 0.6997 \\
$2 X_{0}$ & 0.7591 & 0.7539 & 0.7537 & 0.7546 & 0.7473 \\
$3 X_{0}$ & 0.9960 & 0.8746 & 0.9092 & 0.8743 & 0.8799 \\
$5 X_{0}$ & 1.261 & 1.132 & 1.132 & 1.134 & 1.079 \\
\hline $50 \mathrm{GeV}$ & & & & & \\
$0 X_{0}$ & 1.032 & & & & \\
$1 X_{0}$ & 1.144 & $(1.144)$ & $(1.144)$ & 1.137 & 1.138 \\
$2 X_{0}$ & 1.229 & 1.156 & 1.145 & 1.152 & 1.130 \\
$3 X_{0}$ & 1.582 & 1.281 & 1.392 & 1.313 & 1.314 \\
$5 X_{0}$ & 2.415 & 1.705 & 1.812 & 1.734 & 1.708
\end{tabular}

For values in parentheses, no sensible correction could be determined and therefore none was applied (i.e. the factor was set to 1.0 flat). 
Table $2 b$ : Resolutions (in $\mathrm{GeV}$ ) for different massless-gap correction schemes: Dependence on angle of incidence

$$
\begin{array}{cccc}
\text { no correction } & \text { individual individual } & \text { energy } & \text { overall } \\
\text { mean } & \text { function } & \text { independent } & \text { function }
\end{array}
$$

\begin{tabular}{cccccc}
$10 \mathrm{GeV}$ & & & & \\
$90^{\circ}$ & 0.4711 & 0.4725 & 0.4867 & 0.4556 & 0.4780 \\
$45^{\circ}$ & 0.4926 & 0.4768 & 0.4862 & 0.4724 & 0.4842 \\
$35^{\circ}$ & 0.5544 & 0.5475 & 0.5595 & 0.5474 & 0.5576 \\
$30^{\circ}$ & 0.5941 & 0.5776 & 0.6024 & 0.5733 & 0.5436 \\
\hline $20 \mathrm{GeV}$ & & & & & \\
$90^{\circ}$ & 0.6990 & 0.6943 & 0.7232 & 0.7029 & 0.6996 \\
$45^{\circ}$ & 0.6728 & 0.6739 & 0.6884 & 0.6537 & 0.6784 \\
$35^{\circ}$ & 0.7266 & 0.7272 & 0.7455 & 0.7046 & 0.7101 \\
$30^{\circ}$ & 0.8235 & 0.7618 & 0.8279 & 0.7561 & 0.7254 \\
\hline $50 \mathrm{GeV}$ & & & & & \\
\hline $90^{\circ}$ & 1.067 & 1.064 & N/A & 1.057 & 1.084 \\
$45^{\circ}$ & 1.108 & 1.101 & N/A & 1.067 & 1.073 \\
$35^{\circ}$ & 1.207 & 1.213 & N/A & 1.235 & 1.217 \\
$30^{\circ}$ & 1.311 & $(1.311)$ & N/A & 1.211 & 1.180 \\
& & & & & \\
\hline
\end{tabular}

For values in parentheses, no sensible correction could be determined and therefore none was applied (i.e. the factor was set to 1.0 flat). At $50 \mathrm{GeV}$, no reasonable individual fits were obtained. 
Table 3 : Responses (in $\mathrm{GeV}$ ) for different massless-gap correction schemes: Dependence on thickness of passive material

$$
\begin{array}{cccc}
\text { no correction } & \text { individual individual } & \text { energy } & \text { overall } \\
\text { mean } & \text { function independent function }
\end{array}
$$

$\begin{array}{cccccc}10 \mathrm{GeV} & & & & & \\ 0 X_{0} & 10.01 & & & & \\ 1 X_{0} & 9.845 & 9.991 & 9.904 & 9.850 & 9.995 \\ 2 X_{0} & 9.430 & 9.869 & 9.801 & 9.694 & 9.962 \\ 3 X_{0} & 8.662 & 9.767 & 9.692 & 9.393 & 9.727 \\ 5 X_{0} & 7.351 & 9.621 & 9.622 & 9.248 & 9.334\end{array}$

\section{$20 \mathrm{GeV}$}

$0 X_{0}$

$1 X_{0}$

19.95

$2 X_{0}$

19.83

$3 X_{0}$

19.26

18.02

16.12

$5 X_{0}$

$50 \mathrm{GeV}$

$0 X_{0} \quad 49.90$

$1 X_{0}$

49.78

$2 X_{0}$

49.02

47.17

43.91

$3 X_{0}$

$5 X_{0}$
(19.83)

19.71

19.84

19.94

19.66

19.66

19.61

19.80

19.39

19.52

19.23

19.37

19.33

19.33

19.33

18.98

$(49.78)$
49.44
49.07
48.64

(49.78)

49.81

49.90

49.39

49.59

49.76

48.99

49.23

49.26

48.78

50.02

49.14

For values in parentheses, no sensible correction could be determined and therefore none was applied (i.e. the factor was set to 1.0 flat). 
Table 3 : Responses (in $\mathrm{GeV}$ ) for different massless-gap correction schemes: Dependence on angle of incidence

$$
\begin{array}{cccc}
\text { no correction } & \text { individual individual } & \text { energy } & \text { overall } \\
\text { mean } & \text { function independent } & \text { function }
\end{array}
$$

\begin{tabular}{cccccc}
$10 \mathrm{GeV}$ & & & & \\
$90^{\circ}$ & 9.781 & 9.784 & 9.825 & 9.830 & 10.00 \\
$45^{\circ}$ & 9.534 & 9.605 & 9.590 & 9.659 & 9.970 \\
$35^{\circ}$ & 9.200 & 9.339 & 9.330 & 9.430 & 9.825 \\
$30^{\circ}$ & 8.904 & 9.153 & 9.123 & 9.233 & 9.666 \\
\hline $20 \mathrm{GeV}$ & & & & & \\
$90^{\circ}$ & 19.78 & 19.78 & 19.60 & 19.83 & 19.94 \\
$45^{\circ}$ & 19.40 & 19.49 & 19.48 & 19.55 & 19.75 \\
$35^{\circ}$ & 18.94 & 19.14 & 19.10 & 19.27 & 19.50 \\
$30^{\circ}$ & 18.47 & 18.76 & 18.81 & 18.99 & 19.20 \\
\hline $50 \mathrm{GeV}$ & & & & & \\
$90^{\circ}$ & 49.67 & 49.69 & $\mathrm{~N} / \mathrm{A}$ & 49.76 & 49.88 \\
$45^{\circ}$ & 49.11 & 49.19 & $\mathrm{~N} / \mathrm{A}$ & 49.38 & 49.58 \\
$35^{\circ}$ & 48.46 & 48.50 & $\mathrm{~N} / \mathrm{A}$ & 49.00 & 49.17 \\
$30^{\circ}$ & 47.78 & $(47.78)$ & $\mathrm{N} / \mathrm{A}$ & 48.62 & 48.80 \\
\hline & & & & & \\
\hline & & & & & \\
\hline
\end{tabular}

For values in parentheses, no sensible correction could be determined and therefore none was applied (i.e. the factor was set to 1.0 flat). At $50 \mathrm{GeV}$, no reasonable individual fits were obtained. 

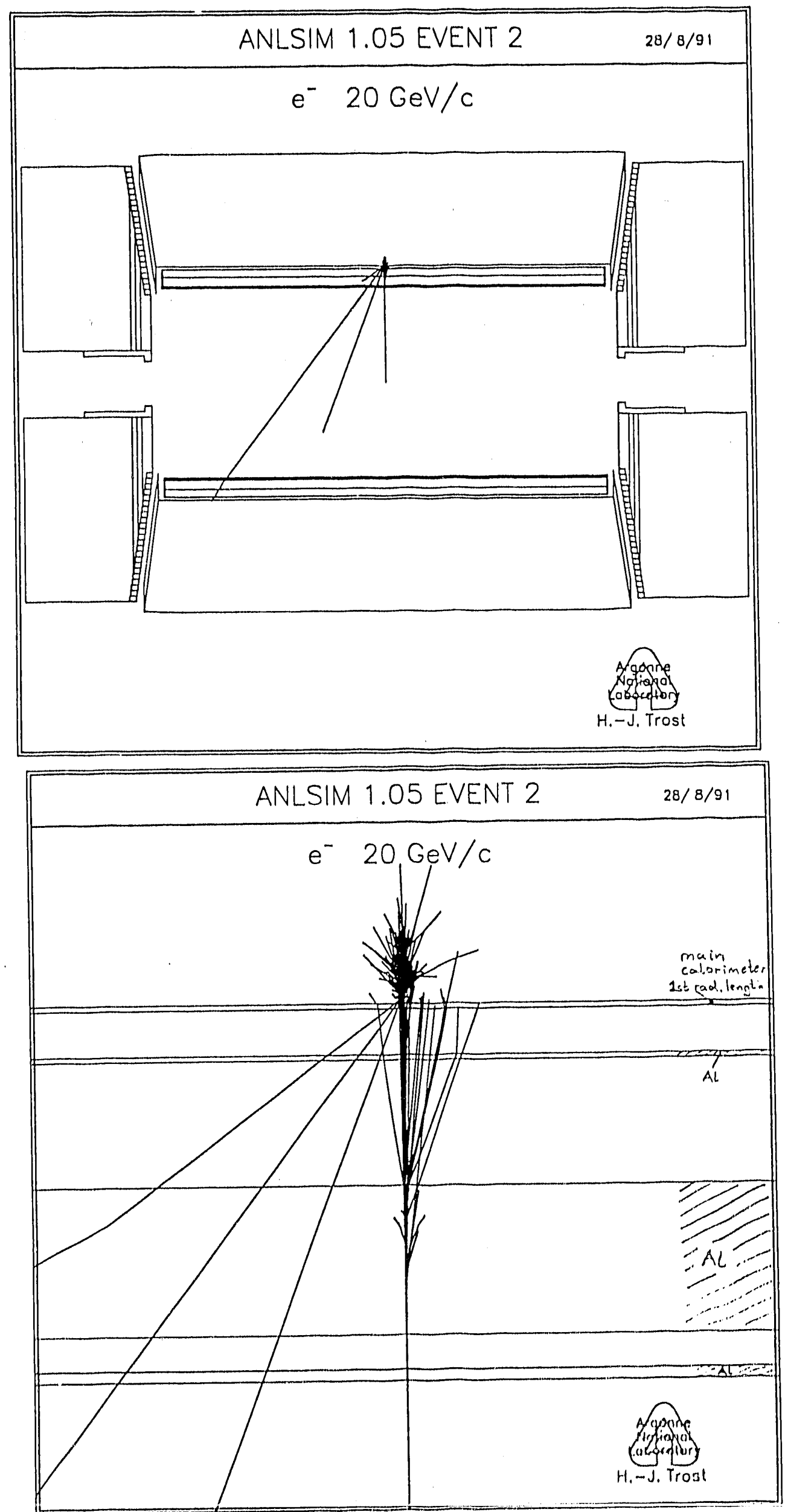

Fig.1 


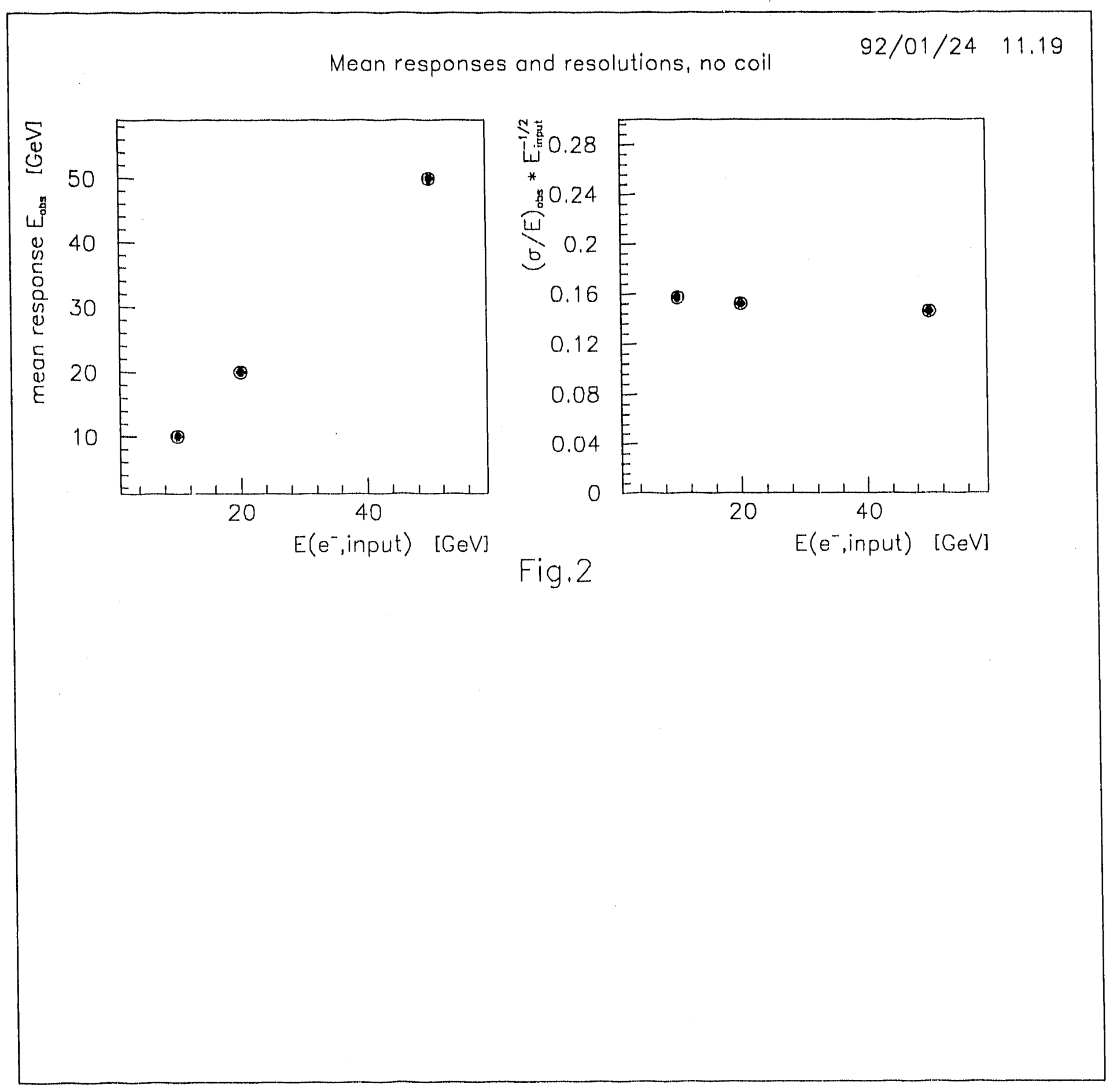




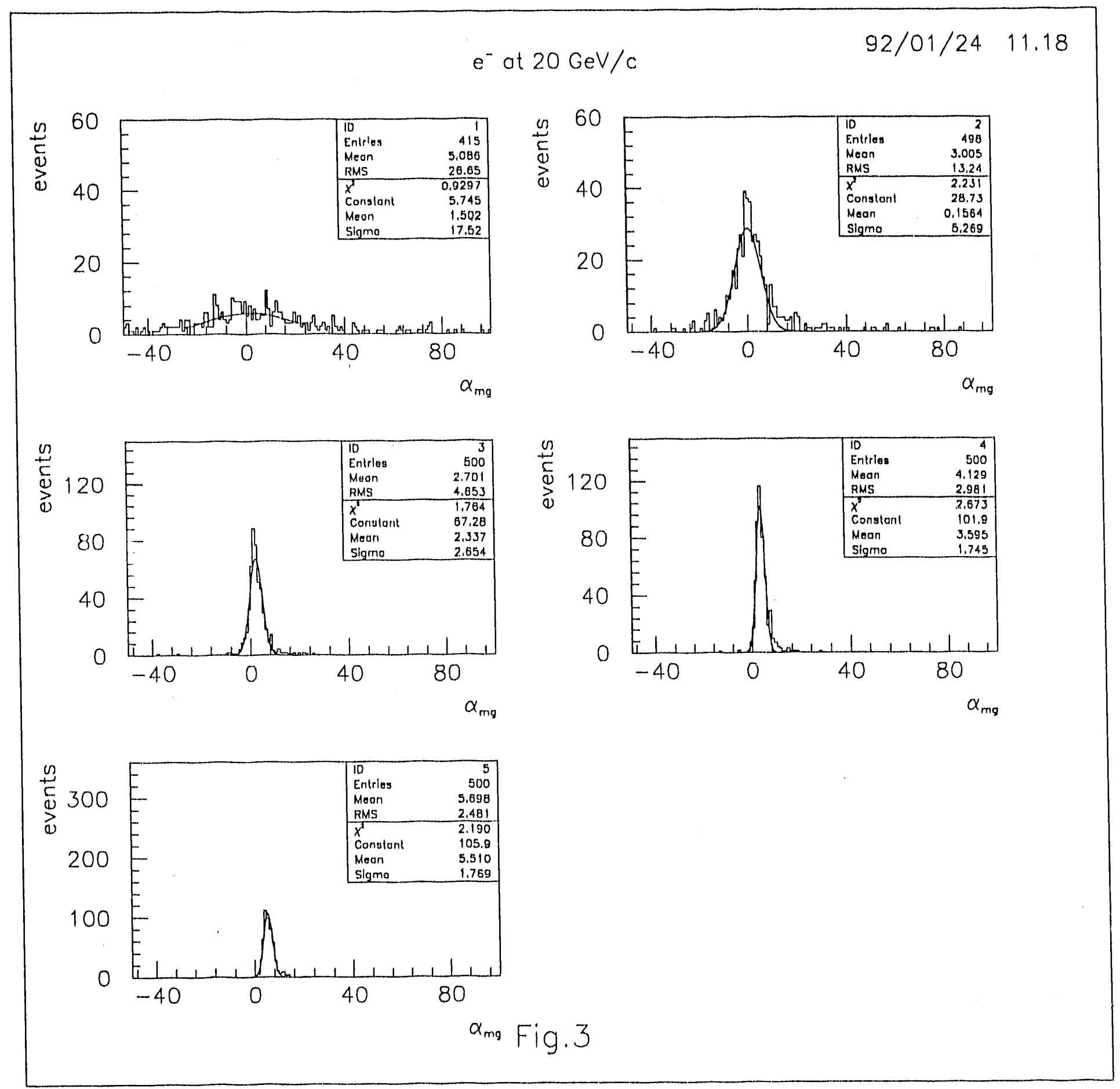




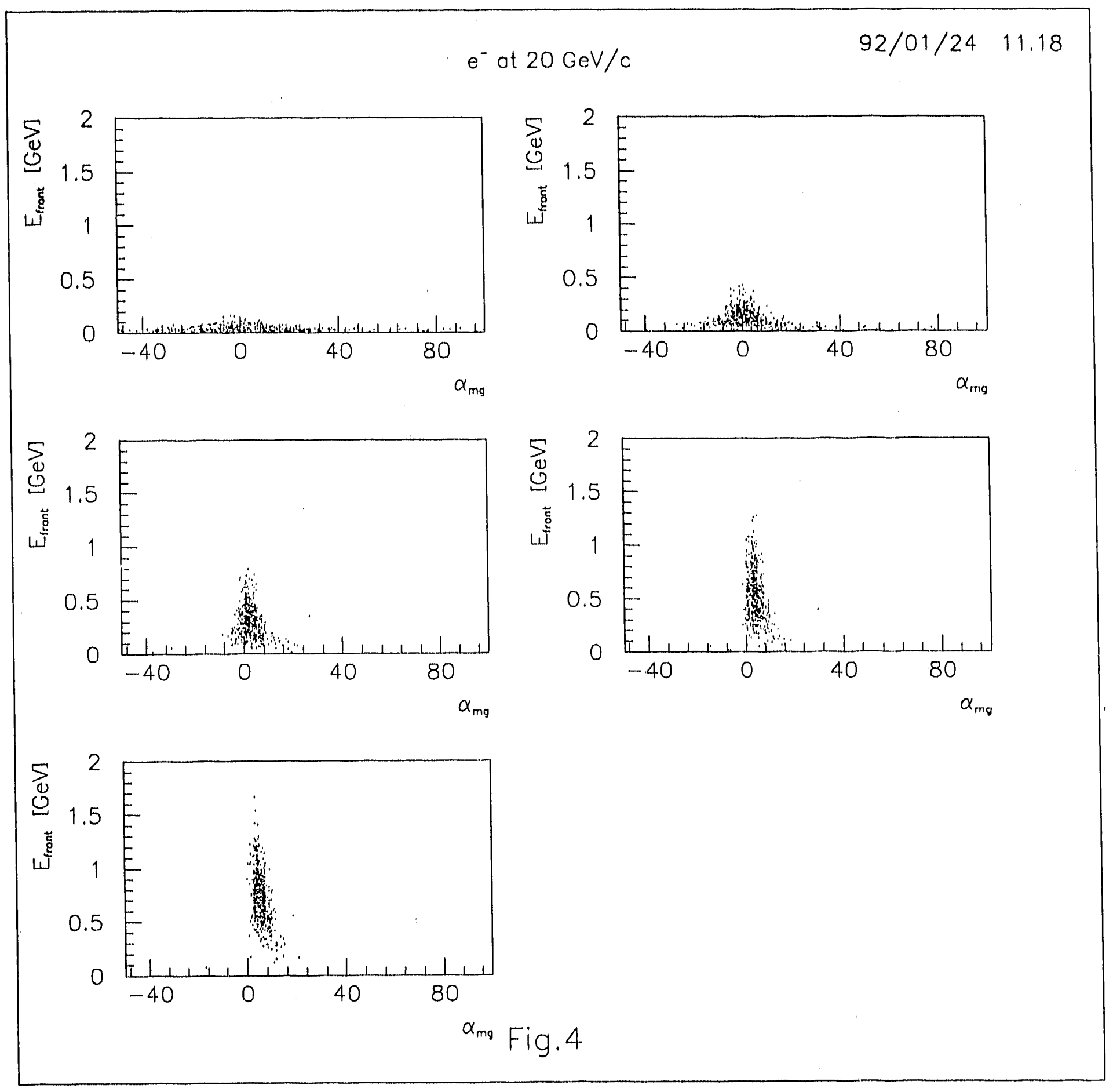


$e^{-}$at $20 \mathrm{GeV} / \mathrm{c}$
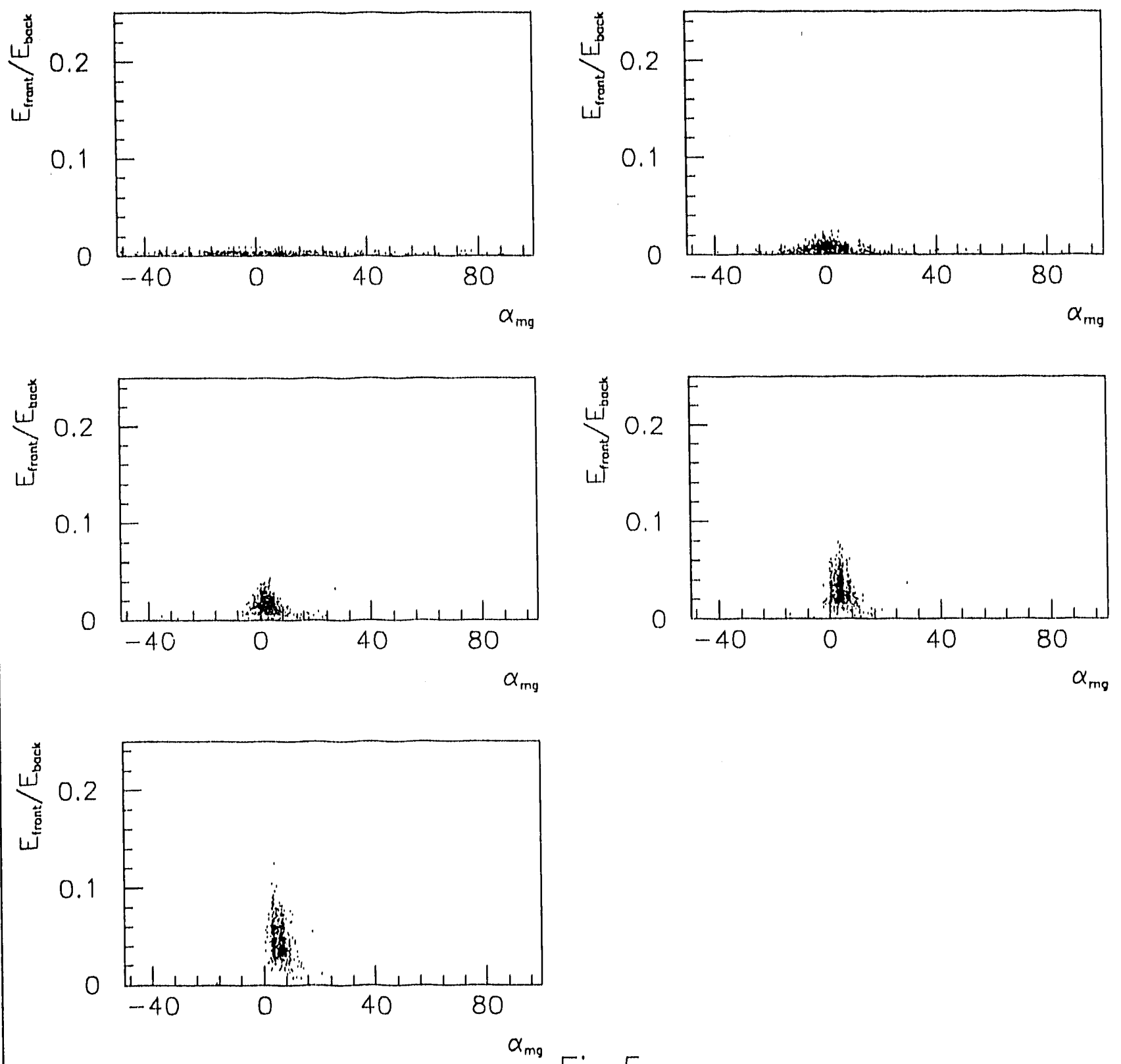

Fig. 5 


$$
e^{-} \text {at } 20 \mathrm{GeV} / \mathrm{c}
$$
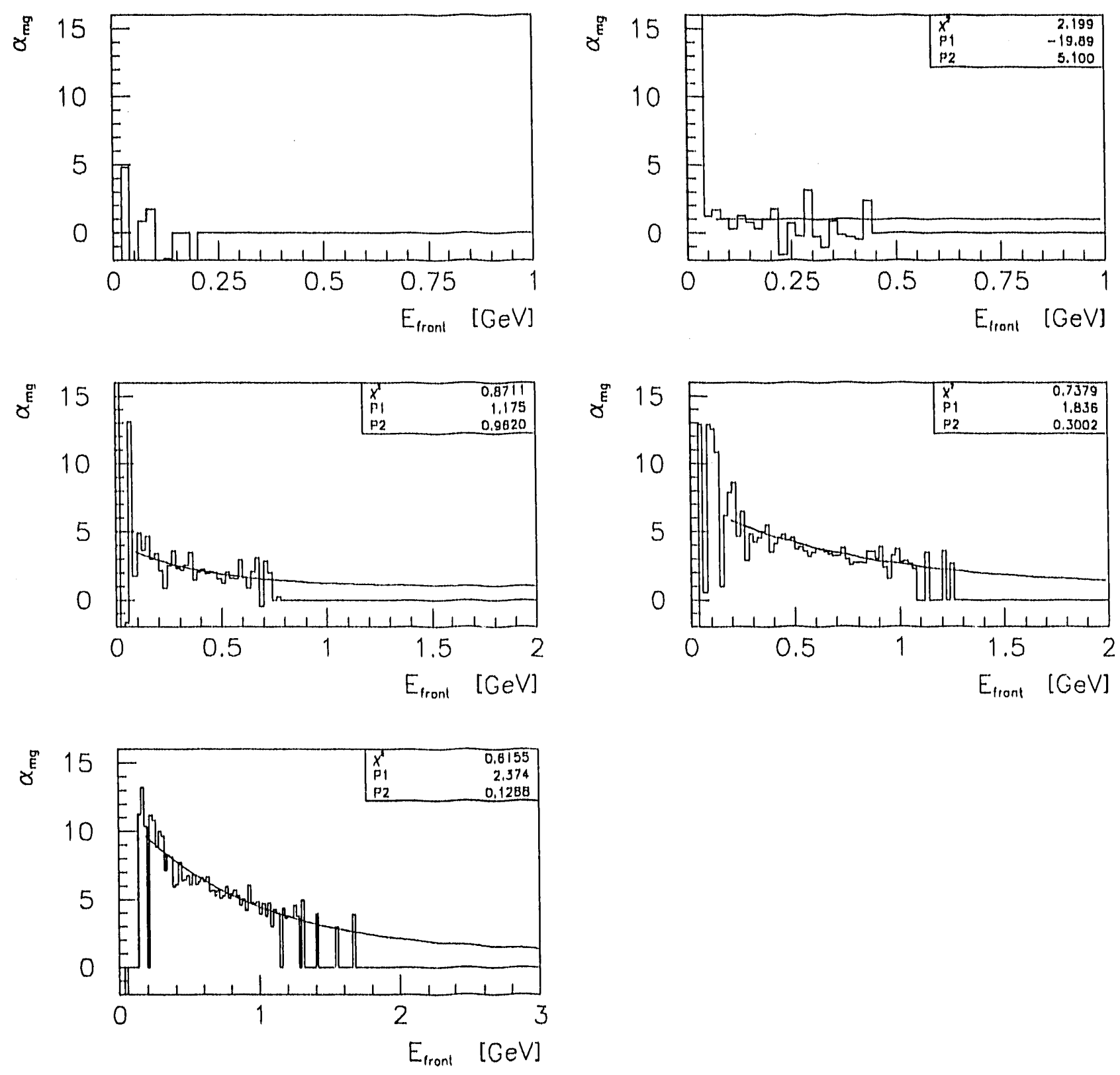

Fig. 6 


$$
\mathrm{e}^{-} \text {at } 20 \mathrm{GeV} / \mathrm{c} \quad 92 / 02 / 04 \quad 16.09
$$
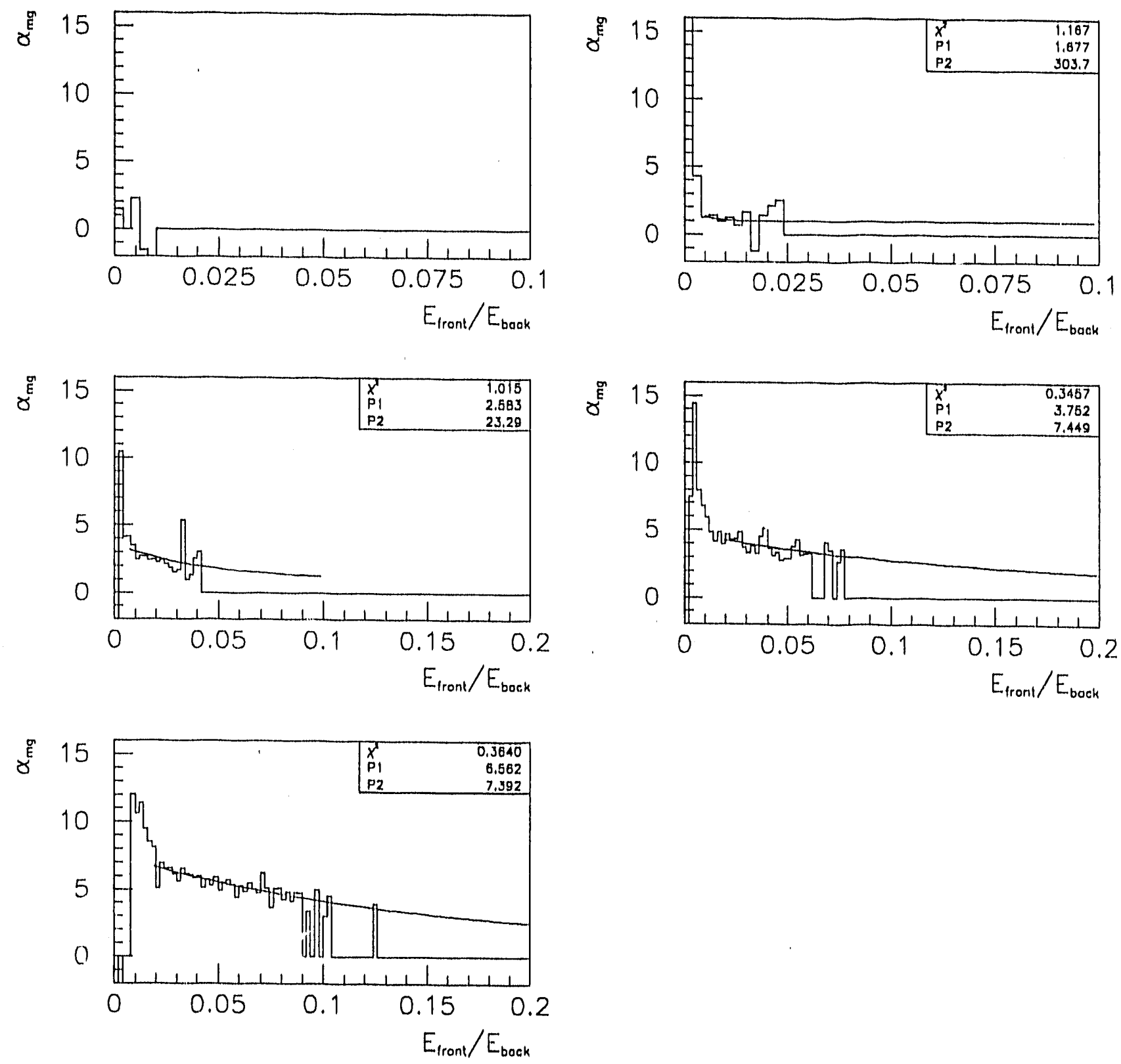

Fig. 7 
Resolution dependence on passive material thickness
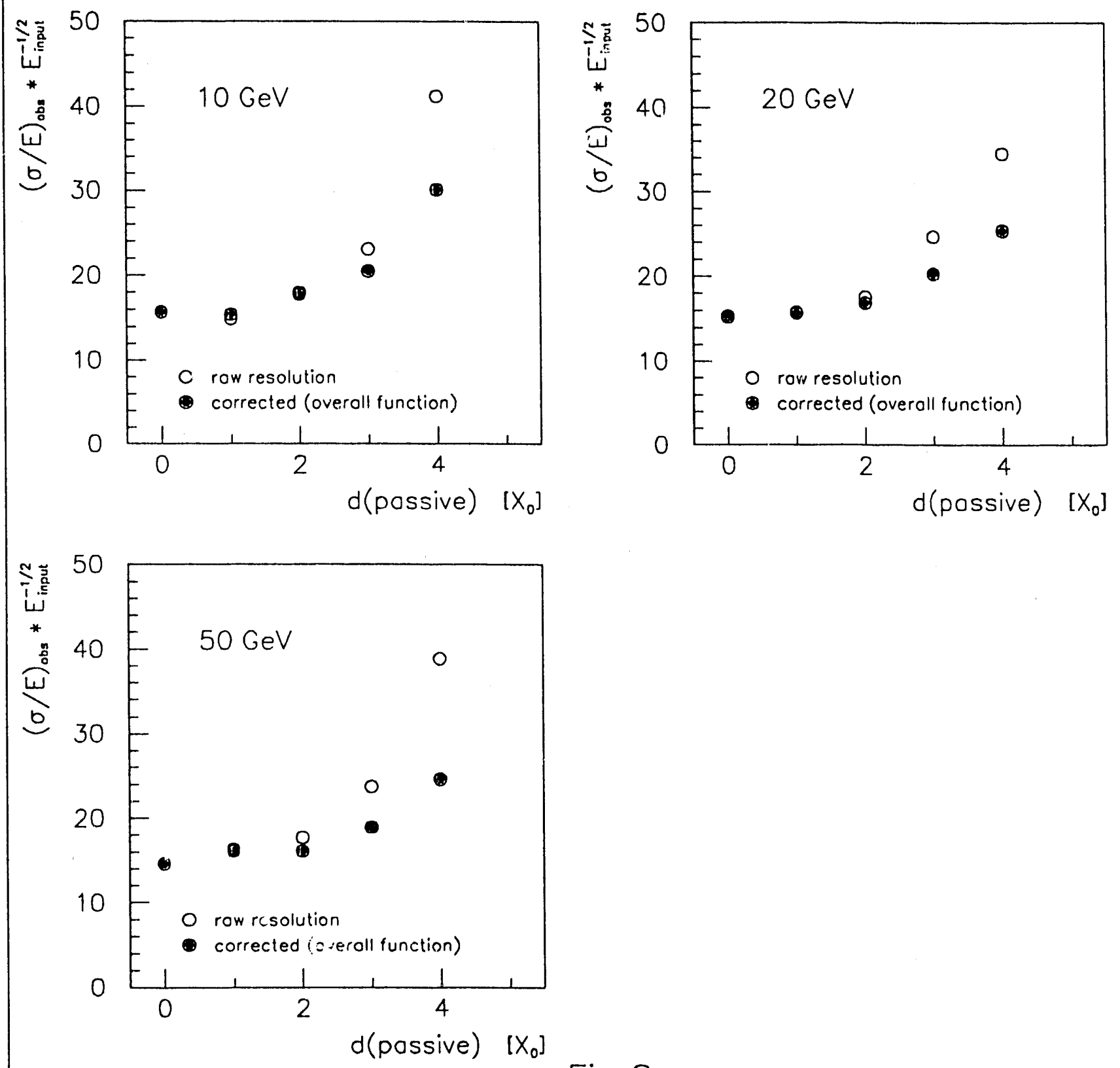

Fig. $8 a$ 
Resolution dependence on angle of incidence
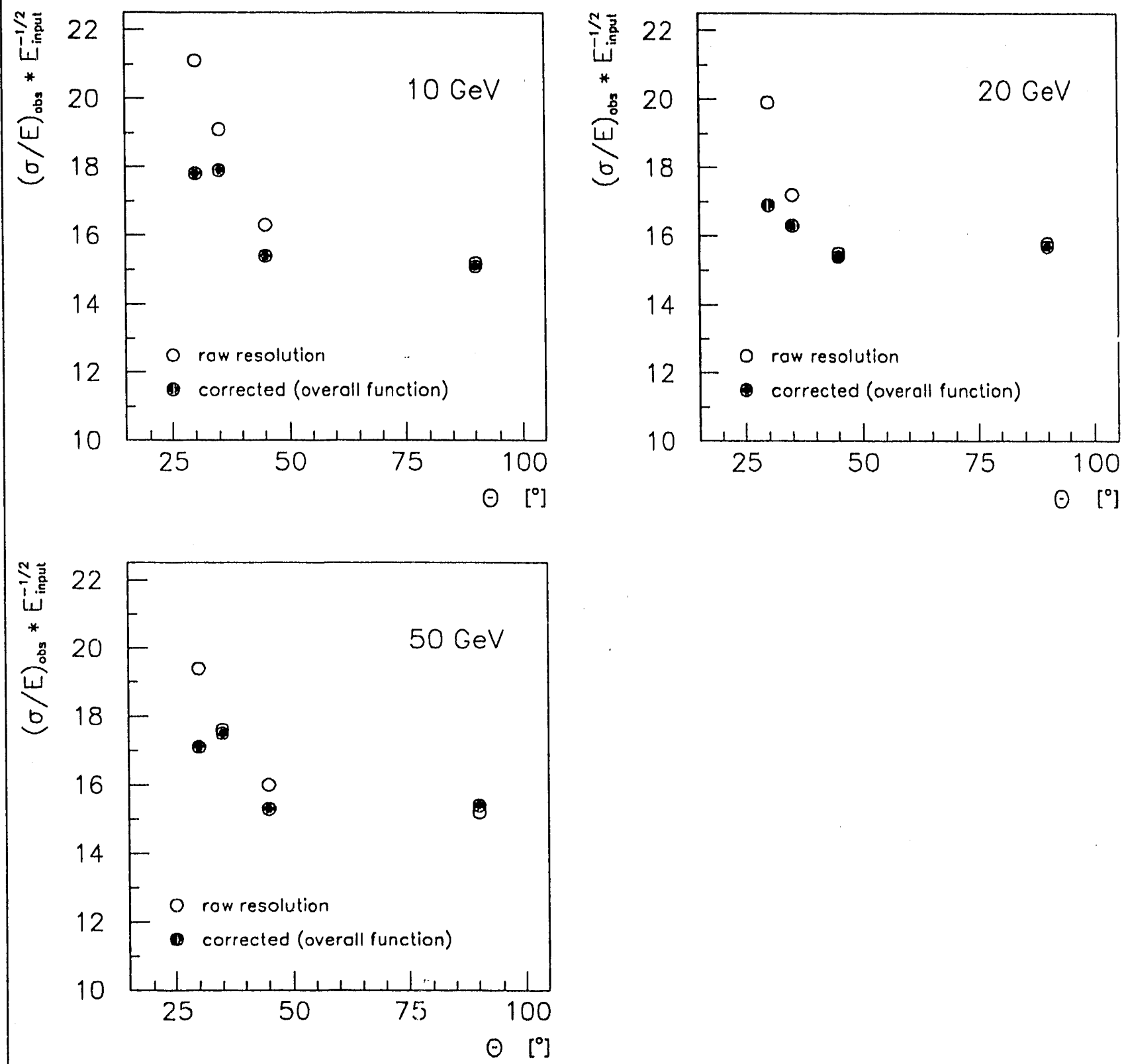

Fig.8b 


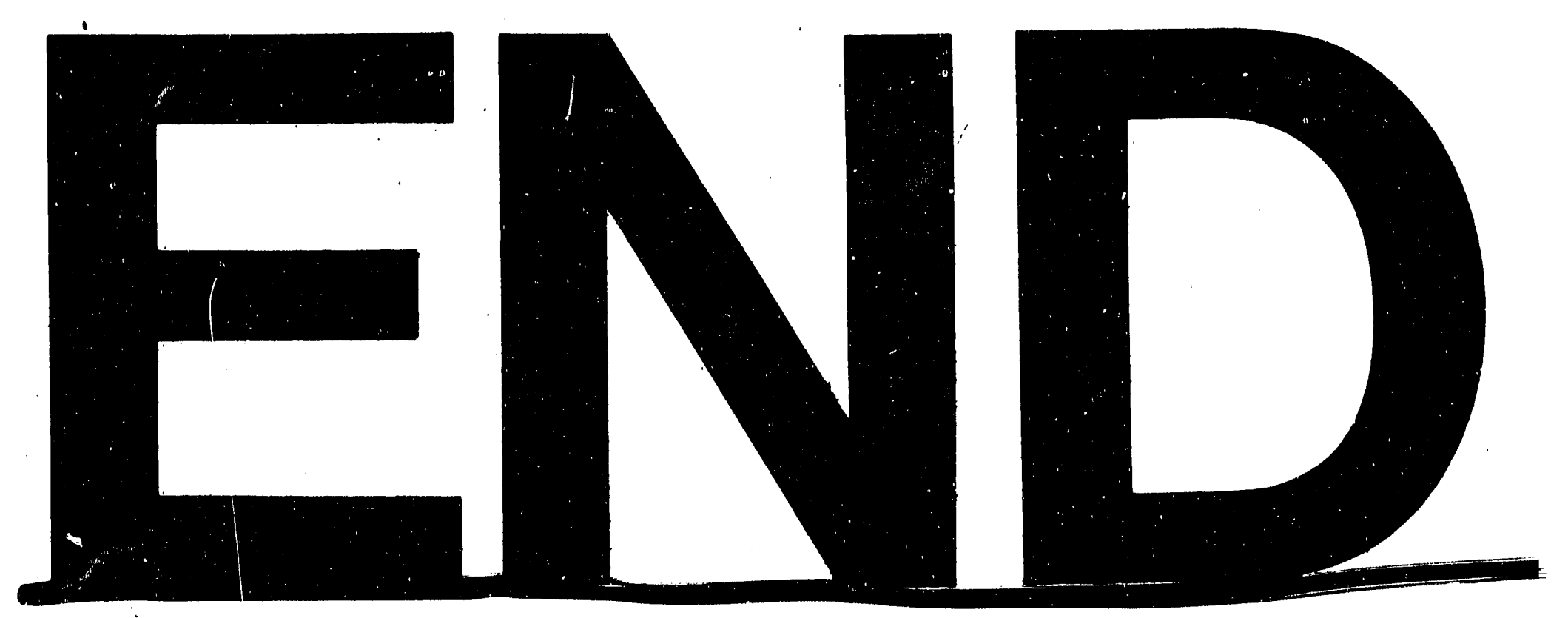

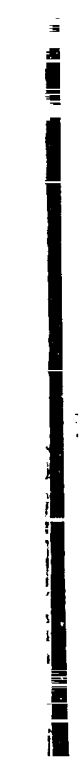

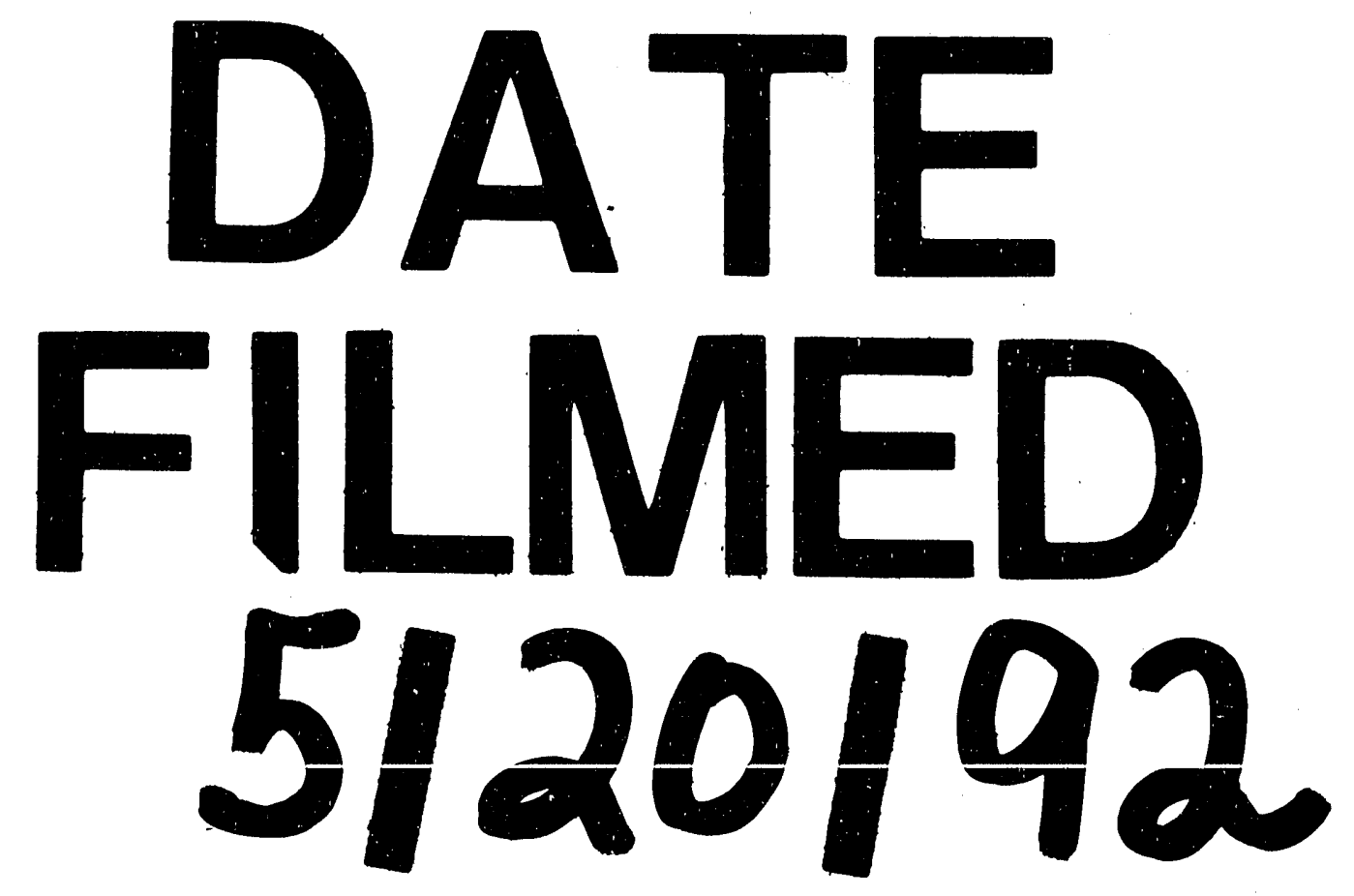


